Joule heating effects on electrokinetic focusing and trapping of particles in constriction microchannels

This article has been downloaded from IOPscience. Please scroll down to see the full text article.

2012 J. Micromech. Microeng. 22075011

(http://iopscience.iop.org/0960-1317/22/7/075011)

View the table of contents for this issue, or go to the journal homepage for more

Download details:

IP Address: 159.226.231.80

The article was downloaded on 11/12/2012 at 23:58

Please note that terms and conditions apply. 


\title{
Joule heating effects on electrokinetic focusing and trapping of particles in constriction microchannels
}

\author{
Junjie Zhu ${ }^{1}$, Sriram Sridharan ${ }^{1}$, Guoqing $\mathrm{Hu}^{2,3}$ and Xiangchun Xuan ${ }^{1,3}$ \\ ${ }^{1}$ Department of Mechanical Engineering, Clemson University, Clemson, SC 29634-0921, USA \\ ${ }^{2}$ LNM, Institute of Mechanics, Chinese Academy of Sciences, Beijing 100190, People's Republic of \\ China \\ E-mail: guoqing.hu@imech.ac.cn and xcxuan@clemson.edu
}

Received 26 February 2012, in final form 26 April 2012

Published 7 June 2012

Online at stacks.iop.org/JMM/22/075011

\begin{abstract}
Joule heating $(\mathrm{JH})$ is a ubiquitous phenomenon in electrokinetic microfluidic devices. Its effects on fluid and ionic species transport in capillary and microchip electrophoresis have been well studied. However, JH effects on the electrokinetic motion of microparticles in microchannels have been nearly unexplored in the literature. This paper presents an experimental investigation of $\mathrm{JH}$ effects on electrokinetic particle transport and manipulation in constriction microchannels under both pure dc and dc-biased ac electric fields. It is found that the JH effects reduce the dielectrophoretic focusing and trapping of particles, especially significant when dc-biased ac electric fields are used. These results are expected to provide a useful guidance for future designs of electrokinetic particle handling microdevices that will avoid $\mathrm{JH}$ effects or take advantage of them.
\end{abstract}

(Some figures may appear in colour only in the online journal)

\section{Introduction}

Joule heating $(\mathrm{JH})$ is a ubiquitous phenomenon in electrokinetic microfluidic devices [1]. It is a volume heat source given by $\sigma_{f} E^{2}$, where $\sigma_{f}$ is the electric conductivity of the liquid and $E$ is the magnitude of the local electric field. JH has been long known to cause temperature rises and gradients first in the fluid and then in the entire device through thermal diffusion [2]. Its effects on fluid electroosmosis and ionic species electrophoresis have been extensively studied in both stand-alone and on-chip microchannels, particularly by the community of capillary and microchip electrophoresis [3-10]. However, JH effects on the electrokinetic particle motion in microchannels have been nearly unexplored in the literature. As electrokinetic means has been frequently used to transport and manipulate synthetic (e.g., glass and polymer beads) and biological (e.g., mammalian and bacterial cells) particles in microfluidic devices $[11,12]$, it is important to

\footnotetext{
${ }^{3}$ Authors to whom any correspondence should be addressed.
}

know what roles JH may play during these particle control processes.

In this work we present our experimental observations of $\mathrm{JH}$ effects on electrokinetic particle transport in constriction microchannels. Such channels have often been used to implement electrokinetic focusing [13, 14], trapping [15-17], concentration [18-20] and separation [21-24] of a variety of particles via dielectrophoresis (DEP) $[25,26]$. However, JH effects in these works have been deliberately suppressed by the use of a low-conductivity fluid and/or a small-magnitude electric field, especially significant when dc-biased ac electric fields are applied [14, 18-21, 23]. Only recently Hawkins and Kirby [27] have developed a 2D numerical model to simulate the effects of $\mathrm{JH}$ on the electrokinetic fluid and particle motion in a microchannel with a constriction in the depth. They found that the action of the electric field on JH-induced fluid conductivity and permittivity gradients gives rise to an electrothermal flow in the constriction region. Such a flow in the form of fluid circulation under appropriate conditions was later experimentally verified by the authors of this paper in a microchannel with a widthwise 
constriction (i.e. a 2D constriction) [28]. Hawkins and Kirby's model also predicted that the electrothermal flow enhances the DEP particle deflection and trapping in almost all cases [27]. Such effects, however, have yet to be experimentally examined.

The objective of this paper is to present the first experimental investigation of $\mathrm{JH}$ effects on electrokinetic particle focusing and trapping in 2D constriction microchannels. It is noted that similar microchannels have also been demonstrated to implement the so-called temperature gradient focusing of ionic species via $\mathrm{JH}$ of the background buffer solution [29-32].

\section{Experiment}

The constriction microchannels used in our experiments were fabricated in poly(dimethylsiloxane) (PDMS) with the standard soft lithography technique. They were formed by bonding the PDMS slab to a regular glass slide after both surfaces were treated by air plasma (PDC-32G, Harrick Scientific, Ossining, NY). The detailed procedure is given elsewhere [14]. The channel is $1 \mathrm{~cm}$ long with a $200 \mu \mathrm{m}$ length constriction in the middle. The widths of the channel and constriction are 400 and $40 \mu \mathrm{m}$, respectively. Two channel depths, 15 and $45 \mu \mathrm{m}$, were used for a comparison of the electrokinetic particle motion under dissimilar JH effects. When all other conditions are maintained similar, JH effects on the $45 \mu \mathrm{m}$ deep channel are expected to be stronger than those in the $15 \mu \mathrm{m}$ deep one. It is because the overall $\mathrm{JH}, \sigma_{f} E^{2} V$, scales with the fluid volume, $V$, inside the microchannel, while the heat dissipation conditions of the PDMS and glass surfaces remain unvaried. We will provide in section 3 an approximate heat transfer analysis of the JH-induced fluid temperature rise in a constriction microchannel (see section 3.2). This experimental design is relevant to the use of deeper channels in electrokinetic microdevices for the purpose of achieving a higher particle throughput $[25,26]$.

The dc and dc-biased ac electric fields were supplied by a function generator (33220A, Agilent Technologies, Santa Clara, CA) in conjunction with a high-voltage amplifier (609E-6, Trek, Medina, NY). The ac field frequency was fixed at $1 \mathrm{kHz}$ in all tests, which was fast enough to cause no net electroosmotic fluid flow and electrophoretic particle motion [12]. Polystyrene particles of $3 \mu \mathrm{m}$ in diameter (Sigma Aldrich, USA) were re-suspended in $5 \mathrm{mM}$ phosphate buffer to a final concentration of $10^{7}$ particles per $\mathrm{ml}$. The electric conductivity of the solution was measured to be $470 \mu \mathrm{S} \mathrm{cm}^{-1}$ at room temperature. The ambient conditions (e.g., room temperature and air flow speed) were maintained for experiments in the two depths of constriction microchannels. Particle motion was monitored using an inverted Nikon microscope (TE2000U, Nikon Instruments, Lewisville, TX), through which videos and images in the constriction region were recorded using a CCD camera (Nikon DS-Qi1Mc). Pressure-driven flow was eliminated by carefully balancing the liquid heights in the two reservoirs prior to each experiment. In addition, the reservoirs were purposely made large $(5 \mathrm{~mm}$ in diameter) in order to minimize the potential influence of backflow on the particle motion.

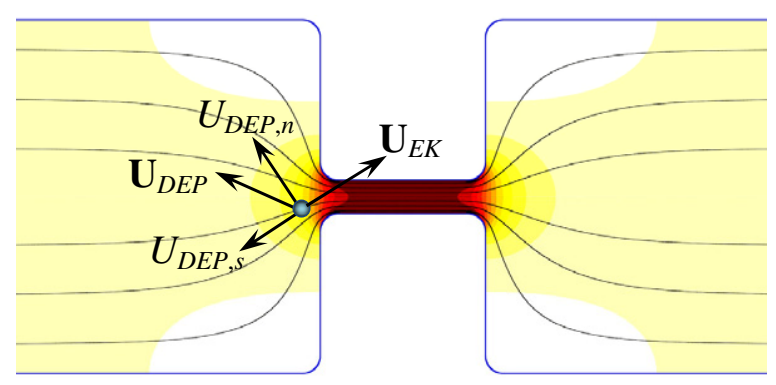

Figure 1. Mechanism for the electrokinetic focusing and trapping of particles in a constriction microchannel. Illustrated is the particle velocity analysis in the absence of Joule heating $(\mathrm{JH})$ effects, where the electrokinetic velocity, $\mathbf{U}_{\mathrm{EK}}$, and the DEP velocity, $\mathbf{U}_{\mathrm{DEP}}$, are each obtained from a force balanced with the hydrodynamic drag [14]. The thin lines and the background color represent the electric field lines and contour (the darker color indicates a larger electric field), respectively.

\section{Theory}

\subsection{Mechanism of electrokinetic particle focusing and trapping}

The mechanism for electrokinetic focusing and trapping of particles in a constriction microchannel by DEP has been covered elsewhere when JH effects are negligible [14, 18]. Here, we only briefly review it without referring to any equation. Due to the non-uniform electric field distribution in the constriction region (see the electric field contour in figure 1), particles experience a negative DEP force under $\mathrm{dc}$ and low-frequency ac electric fields [25, 26], inducing a

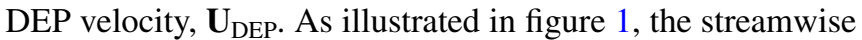
component of this velocity, $U_{\mathrm{DEP}, \mathrm{s}}$, opposes the electrokinetic particle motion, $\mathbf{U}_{\mathrm{EK}}$. As the former increases more quickly with the electric field than does $\mathbf{U}_{\mathrm{EK}}$, a stagnation zone can be formed for particles in front of the constriction leading to particle trapping. Moreover, the cross-stream component of the DEP velocity, $U_{\mathrm{DEP}, \mathrm{n}}$, deflects particles to the channel center (see figure 1). The consequence is 2D particle focusing in the horizontal plane, which can be enhanced by increasing the electric field as well $[13,14]$. As ac electric fields do not contribute to $\mathbf{U}_{\mathrm{EK}}$, the use of dc-biased ac electric fields can strengthen the particle trapping and focusing [14, 18-21, 23].

However, when a large electric field (for manipulating small particles such as bacteria $[16,20]$ ) and/or a highly conductive fluid (for manipulating mammalian cells $[18,22,24]$ ) are needed, JH effects become significant. Electrothermal flow can be induced by the interaction of the electric field and JH-induced fluid conductivity and permittivity gradients in the channel constriction region [27]. This flow is superimposed on the electrokinetic flow and not streamwise, and may even involve fluid circulations as demonstrated in [28]. Therefore, $\mathrm{JH}$ is expected to have a direct impact on the above-analyzed electrokinetic focusing and trapping of particles in constriction microchannels.

\subsection{Approximate heat transfer analysis of JH-induced fluid temperature rise}

Figure 2 shows the cross-sectional schematic of the experimental microdevice (not to scale). As it has a much 


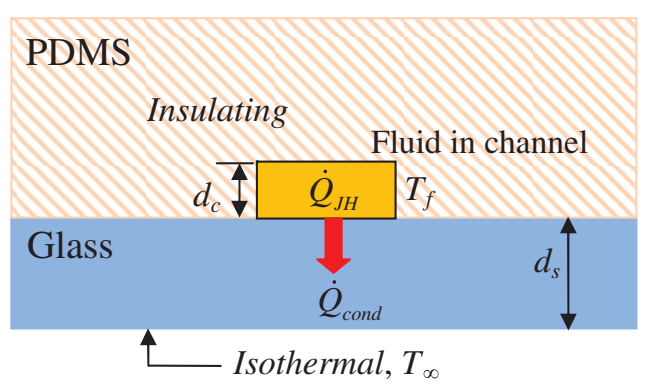

Figure 2. Cross-sectional schematic (not to scale) of the experimental microdevice showing heat transfer approximations.

lower thermal conductivity than glass $\left(1.4 \mathrm{~W}(\mathrm{~m} \mathrm{~K})^{-1}\right)$, PDMS $\left(0.18 \mathrm{~W}(\mathrm{~m} \mathrm{~K})^{-1}\right)$ can be assumed to be thermally insulating. Then, if we neglect the axial temperature variations which is valid except near the constriction $[27,28]$ and the end-channel reservoirs [33, 34], JH of the fluid at any cross-section of the microchannel, $\dot{Q}_{\mathrm{JH}}=\sigma_{f} E^{2} w d_{c}$, is dissipated only through the glass substrate via an approximately $1 \mathrm{D}$ heat conduction, $\dot{Q}_{\text {cond }}=k_{s}\left(T_{f}-T_{\infty}\right) w / d_{s}$. In these two expressions, $w$ is the width of the microchannel, $d_{c}$ is the channel depth, $k_{s}$ is the thermal conductivity of the glass substrate with a thickness $d_{s}, T_{f}$ is the mean fluid temperature over the microchannel cross-section, and $T_{\infty}$ is the temperature of the lower surface of the glass substrate that is in contact with a working stage in experiment and can thus be assumed isothermal. As such, the fluid temperature rise at a steady state is given by $T_{f}-T_{\infty}=\sigma_{f} E^{2} d_{c} d_{s} / k_{s}$, which is consistent with the scaling analysis from Ramos et al [35]. This approximate heat transfer analysis indicates that JH-induced fluid temperature elevation increases with the microchannel depth $d_{c}$ if all other conditions remain unvaried. Note that the temperature dependence of electrical and thermophysical properties (i.e. $\sigma_{f}$ and $k_{s}$ ) is not considered in the above approximate analysis.

\section{Results and discussion}

\subsection{Electrokinetic particle focusing under pure dc electric fields}

Figure 3 compares the streak images for the dc electrokinetic motion of $3 \mu \mathrm{m}$ particles in the $15 \mu \mathrm{m}$ (left column) and $45 \mu \mathrm{m}$ (right column) deep constriction microchannels. These images were obtained by superimposing a sequence of steadystate images taken at least $1 \mathrm{~min}$ after the electric voltage was turned on. The applied voltage drop was varied from $200 \mathrm{~V}$ (a) to $400 \mathrm{~V}$, (b) $600 \mathrm{~V}$ and (c) $800 \mathrm{~V}$ (d), corresponding to an average dc electric field from 200 to $800 \mathrm{~V} \mathrm{~cm}^{-1}$. All the other parameters were fixed in the tests between the two depths of microchannels.

Similar to what has been demonstrated previously $[13,14,18]$, particles in all cases are pushed away from the corners of the constriction and deflected toward the channel midplane by the induced negative DEP. The consequence is a squeezed particle stream flowing in the channel center region downstream of the constriction, in contrast to the uniform particle distribution covering almost the entire channel width upstream. Such DEP focusing increases with the rise of the
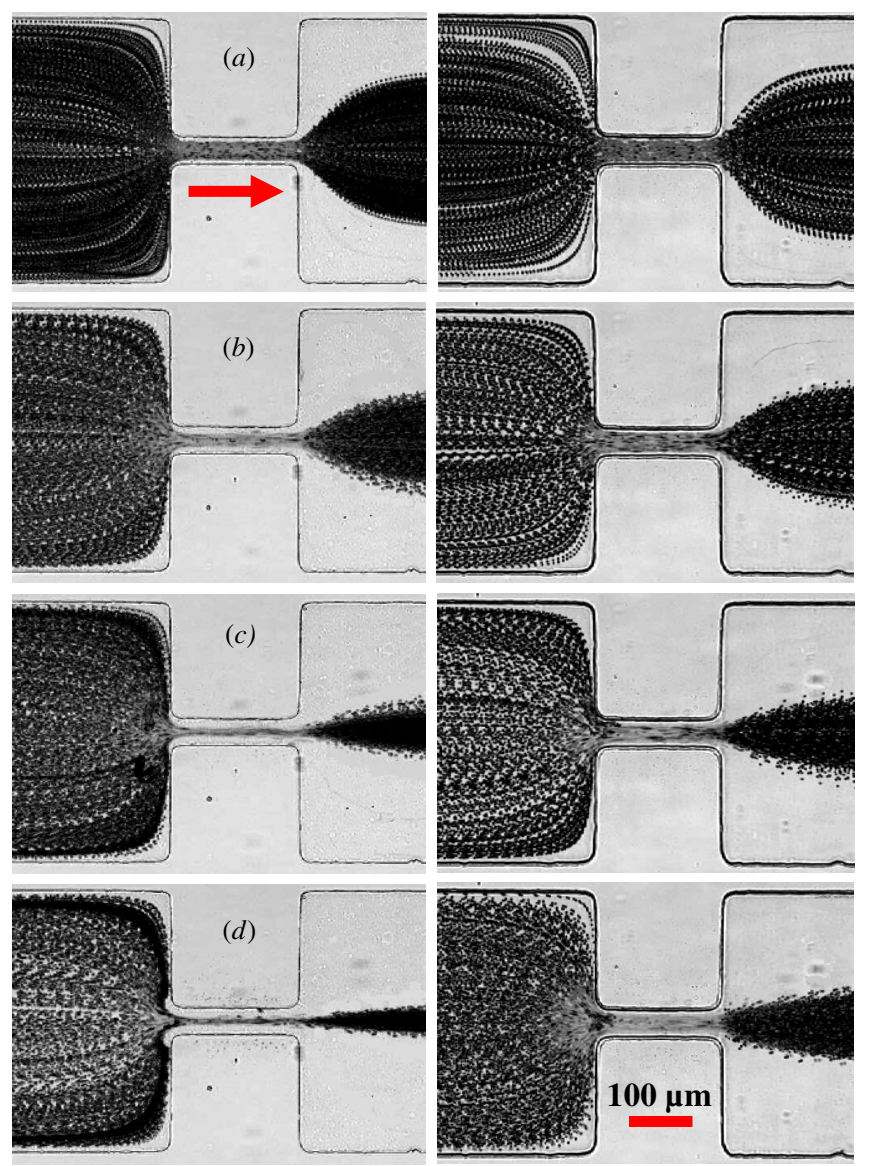

Figure 3. Superimposed images illustrating the effects of JH on the electrokinetic focusing of $3 \mu \mathrm{m}$ particles through $15 \mu \mathrm{m}$ (left column) and $45 \mu \mathrm{m}$ (right column) deep microchannel constrictions under various pure dc electric fields (average value over the channel length): (a) $200 \mathrm{~V} \mathrm{~cm}^{-1}$, (b) $400 \mathrm{~V} \mathrm{~cm}^{-1}$, (c) $600 \mathrm{~V} \mathrm{~cm}^{-1}$ and (d) $800 \mathrm{~V} \mathrm{~cm}^{-1}$. The block arrow in $(a)$ indicates the particle moving direction.

electric field in both depths of microchannels. However, the width of the focused particle stream is larger in the $45 \mu \mathrm{m}$ deep channel (right column of figure 3) than in the $15 \mu \mathrm{m}$ deep one (left column of figure 3 ) at each dc field tested. This is attributed to the stronger JH effects on the deeper channel as analyzed in section 3.2. In other words, JH effects compromise the electrokinetic particle focusing in microchannels with a widthwise constriction, which is opposite to Hawkins and Kirby's numerical prediction of enhancement in their microchannel with a depthwise constriction [27]. In the latter, a single hurdle is located on the bottom channel wall, forming a constriction in the channel depth. The induced DEP deflects particles toward the top channel wall, which is found to be enhanced by $\mathrm{JH}$ and electrothermal effects in Hawkins and Kirby's 2D numerical model. We are uncertain what causes this controversy. A 3D numerical model is currently under development by the authors, which is expected to offer a fundamental understanding of the real transport phenomena in each of these two devices.

There is one issue in the current experiment which can cause an unequal influence on the particle focusing in the two depths of microchannels. As the channel depth increases from 

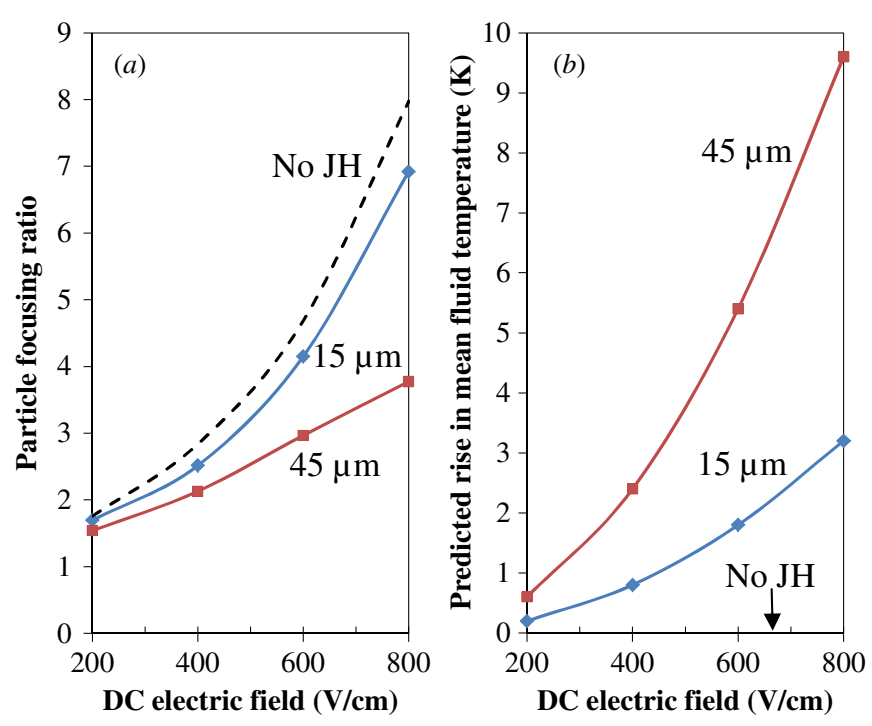

Figure 4. Comparisons of the experimentally measured particle focusing ratios $(a)$ and the theoretically predicted fluid temperature rises $(b)$ between the 15 and $45 \mu \mathrm{m}$ deep constriction microchannels under various dc electric fields. The dashed line in $(a)$ is the numerical prediction of the particle focusing ratio in the absence of $\mathrm{JH}$ effects, which is independent of the microchannel depth. The symbols are the experimental $(a)$ and theoretical $(b)$ data, and the solid lines are used only to guide the eyes.

15 to $45 \mu \mathrm{m}$, the surface area of the channel walls composed of PDMS rather than glass increases. This will change the average electroosmotic velocity and in turn the electrokinetic particle motion [36]. However, since the zeta potential of the PDMS surface is not significantly different from that of the glass surface [37], and moreover, the increase of the channel wall surface area is still relatively small as compared to the overall wall surface area $(30 \mu \mathrm{m}$ versus $830 \mu \mathrm{m}$ per unit channel length of the two microchannels), we estimate that this issue should have only a subtle effect on the electrokinetic particle transport in our experiments.

To quantify the $\mathrm{JH}$ effects on the electrokinetic particle motion, we define particle focusing ratio as the ratio of particle stream width before and after the constriction. Figure 4(a) shows the comparison of this focusing ratio in the 15 and $45 \mu \mathrm{m}$ deep microchannels under the application of various dc electric fields. We see that the focusing ratio in the $15 \mu \mathrm{m}$ deep channel increases much faster than that in the $45 \mu \mathrm{m}$ one when the dc field increases. At low dc fields (e.g., 200 and $400 \mathrm{~V} \mathrm{~cm}^{-1}$ ), the particle focusing ratio is comparable between the two depths of microchannels (see also figures $3(a),(b))$ as the $\mathrm{JH}$ effects are still relatively weak in both channels. At higher dc fields (e.g., 600 and $800 \mathrm{~V} \mathrm{~cm}^{-1}$ ), however, JH effects become significant in the $45 \mu \mathrm{m}$ deep microchannel, while remaining moderate or insignificant in the $15 \mu \mathrm{m}$ deep channel. The result is a substantially reduced particle focusing ratio in the deeper channel (see also figures $3(c)$ and $(d)$ ). This analysis is supported by the theoretically predicted fluid temperature rises in the two microchannels as seen in figure $4(b)$. To obtain the theoretical curves, we used the following parameters in the approximate heat transfer analysis (see section 3.2): the electric conductivity of the fluid is $\sigma_{f}=$ $0.047 \mathrm{~S} \mathrm{~m}^{-1}$, and the thermal conductivity and thickness of the glass substrate are $k_{s}=1.4 \mathrm{~W}(\mathrm{~m} \mathrm{~K})^{-1}$ and $d_{s}=1 \mathrm{~mm}$, respectively.

Also illustrated in figure $4(a)$ is the numerically predicted curve of the particle focusing ratio (dashed line) when $\mathrm{JH}$ effects are not considered. It was obtained using the model developed in COMSOL ${ }^{\circledR}$ by our group $[14,18]$. For this modeling, the electrokinetic mobility of $3 \mu \mathrm{m}$ particles was measured at a $100 \mathrm{~V} \mathrm{~cm}^{-1} \mathrm{dc}$ field to minimize $\mathrm{JH}$ effects, and was found to be $0.83\left(\mu \mathrm{m} \mathrm{s}^{-1}\right) /\left(\mathrm{V} \mathrm{cm}^{-1}\right)$. The correction factor was set as 0.8 to account for the influence of particle size on the DEP velocity, which is consistent with our earlier studies [38]. Twenty evenly distributed points over the width of the microchannel at the inlet were chosen to represent the initial positions of particles, and so the particle stream width before the constriction was equal to the microchannel width. The trajectory of each of these particles along the constriction microchannel was tracked according to the combined electrokinetic and DEP velocity, i.e. $\mathbf{U}_{\mathrm{EK}}+\mathbf{U}_{\mathrm{DEP}}$. The particle stream width after the constriction was directly measured from the predicted particle trajectories, from which the particle focusing ratio could be calculated.

One can see in figure 4(a) that the experimentally obtained particle focusing ratios (solid lines with symbols) in the two microchannels are both smaller than the numerical prediction (dashed line) due to JH effects. Moreover, the deviations increase with the increase of the electric field, especially significant in the $45 \mu \mathrm{m}$ deep channel due to the stronger $\mathrm{JH}$ effects therein. This trend is consistent with the theoretically predicted fluid temperature rises in the two microchannels as shown in figure $4(b)$. It is important to note that the particle DEP induced in the current 2D microchannel constriction should be independent of the channel depth [13-19, 22-26]. Therefore, the focusing effectiveness should remain similar between the two tested microchannels when $\mathrm{JH}$ effects are insignificant as at $200 \mathrm{~V} \mathrm{~cm}^{-1}$ (see figures $3(a)$ and 4(a)).

\subsection{Electrokinetic particle focusing and trapping under dc-biased ac electric fields}

Figure 5 shows the streak images of $3 \mu \mathrm{m}$ particles in the $15 \mu \mathrm{m}$ (left column) and $45 \mu \mathrm{m}$ (right column) deep constriction microchannels under dc-biased ac electric fields. The total root-mean-square (RMS) value of the dc and ac fields was fixed at $500 \mathrm{~V} \mathrm{~cm}^{-1}$. The ac field frequency was fixed at $1 \mathrm{kHz}$. At a $500 \mathrm{~V} \mathrm{~cm}^{-1}$ pure dc field (see figure $5(a)$ ), the particle images are consistent with those illustrated in figure 3 (see (b) for $400 \mathrm{~V} \mathrm{~cm}^{-1}$ and (c) for $600 \mathrm{~V} \mathrm{~cm}^{-1}$ ) for both depths of microchannels. With the inclusion and increase of the ac field (see figures $5(b)-(d)$ ), particle focusing is enhanced in both channels as the ac field contributes to DEP but not to the electrokinetic motion. However, the particle stream width in the $45 \mu \mathrm{m}$ deep microchannel (right column of figure 5) is apparently larger than that in the $15 \mu \mathrm{m}$ deep channel due to the greater $\mathrm{JH}$ effects in the former. This observation is consistent with that for the electrokinetic particle motion under pure dc fields in figure 3 . Particularly at $50 \mathrm{~V} \mathrm{~cm}^{-1} \mathrm{dc} / 450 \mathrm{~V} \mathrm{~cm}^{-1} \mathrm{ac}$ 

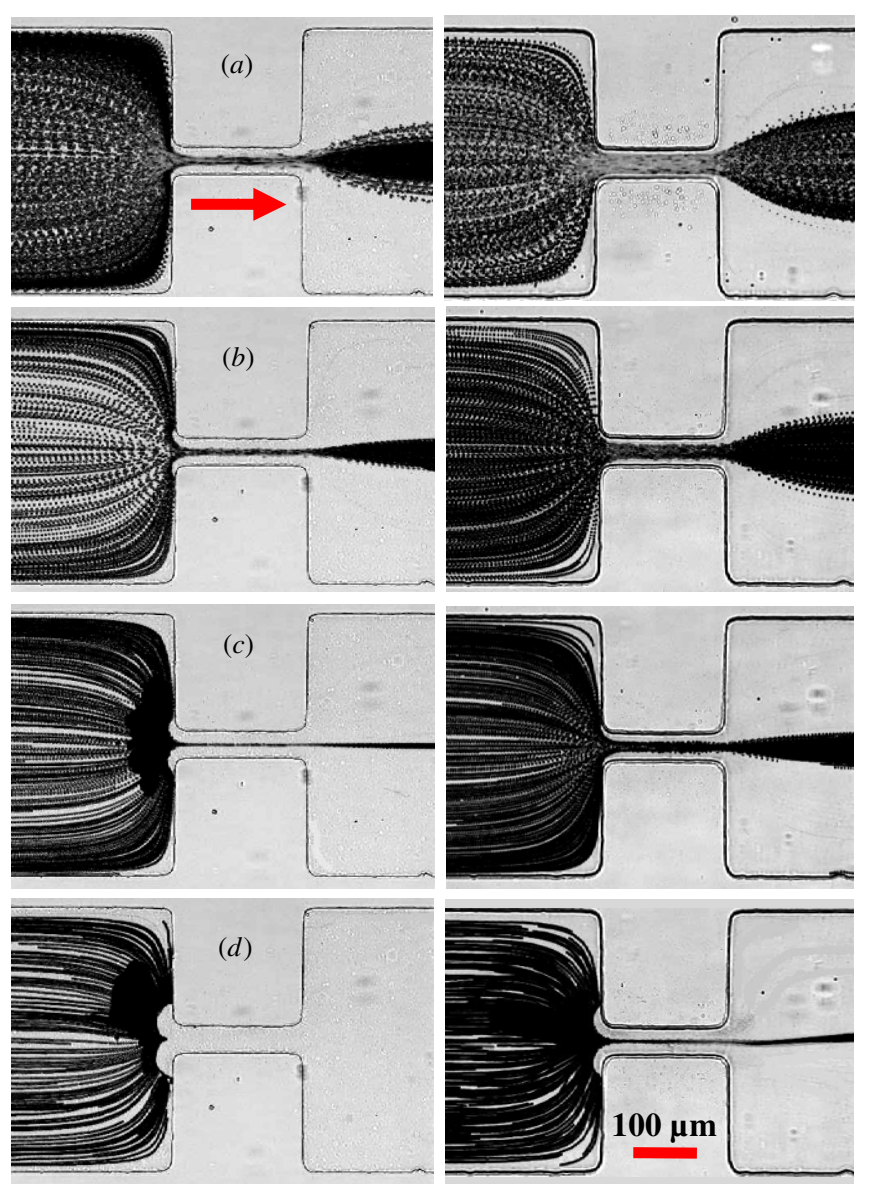

Figure 5. Superimposed images illustrating the effects of $\mathrm{JH}$ on the electrokinetic motion of $3 \mu \mathrm{m}$ particles through $15 \mu \mathrm{m}$ (left column) and $45 \mu \mathrm{m}$ (right column) deep microchannel constrictions under various dc-biased ac electric fields (in units of $\mathrm{V} \mathrm{cm}^{-1}$ ): (a) $500 \mathrm{dc} / 0 \mathrm{AC}$ (i.e. the ac to dc ratio is $\alpha=0) ;(b) 166 \mathrm{dc} / 334 \mathrm{ac}(\alpha=$ 2); (c) $100 \mathrm{dc} / 400$ ac $(\alpha=4)$; (d) $50 \mathrm{dc} / 450$ ac $(\alpha=9)$. The block arrow in $(a)$ indicates the particle moving direction.

(RMS), which has an ac to dc ratio of $\alpha=9$, particles are trapped and concentrated in front of the constriction in the $15 \mu \mathrm{m}$ deep microchannel (see figure $5(d)$, left) due to the induced negative DEP that overcomes the electrokinetic particle motion. In contrast, particles can still pass through the constriction in the $45 \mu \mathrm{m}$ deep channel (see figure $5(d)$, right).

The comparison of particle focusing ratios in the 15 and $45 \mu \mathrm{m}$ deep constriction microchannels under dc-biased ac electric fields is shown in figure 6. The discrepancy between these two ratios quickly increases with increasing ac to dc field ratio, $\alpha$, indicating a greater impact of $\mathrm{JH}$ effects on the electrokinetic particle motion under dc-biased ac electric fields as compared to pure dc fields. This is interesting because the $\mathrm{JH}$ induced fluid temperature rise is estimated as $T_{f}-T_{\infty}=\sigma_{f} E_{\mathrm{dc}}^{2}\left(1+\alpha^{2}\right) d_{c} d_{s} / k_{s}$, which is approximately 1 and $3 \mathrm{~K}$ in the 15 and $45 \mu \mathrm{m}$ deep microchannels, respectively, when the ac to dc field ratio, $\alpha$, is varied. We speculate that it is the JH-induced electrothermal flows that lead to the observed difference in particle focusing and trapping in between the two channels at various values of $\alpha$. This is based on the fact that electrothermal flows grow with the increase of $\alpha$, which has been recently demonstrated by the authors [28]. In addition,

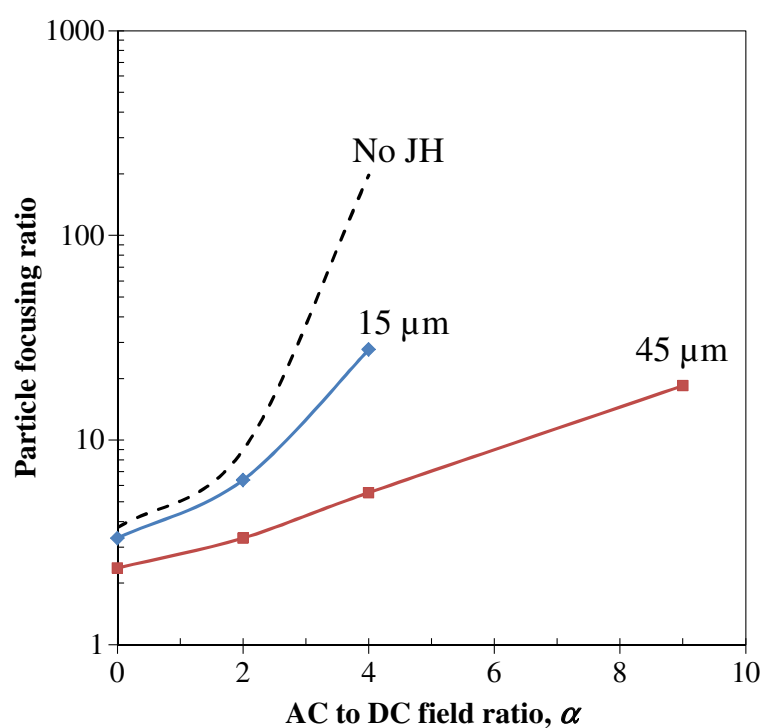

Figure 6. Comparison of the experimentally measured particle focusing ratios between the 15 and $45 \mu \mathrm{m}$ deep constriction microchannels under dc-biased ac electric fields. The total magnitude of the ac and dc electric fields is $500 \mathrm{~V} \mathrm{~cm}^{-1}$ while the ac to $\mathrm{dc}$ field ratio, $\alpha$, is varied. The dashed line is the numerical prediction of the particle focusing ratio in the absence of $\mathrm{JH}$ effects, which is independent of the microchannel depth. The symbols are the experimental data, and the solid lines are used only to guide the eyes.

similar to what we see in figure 4(a), the particle focusing ratios in both depths of microchannels under dc-biased ac electric fields are also smaller than the numerical prediction without consideration of JH (dashed line in figure 6). This observation further validates the statement we made earlier that $\mathrm{JH}$ effects reduce the electrokinetic particle focusing and trapping in 2D-constriction microchannels.

\section{Conclusions}

We have performed an experimental study of Joule heating $(\mathrm{JH})$ effects on the electrokinetic particle transport and manipulation in rectangular microchannels with a widthwise constriction in the middle of the channel length. Through the use of microchannels of two different depths as well as the comparison with the numerical prediction in the absence of $\mathrm{JH}$ effects, we demonstrate that the dielectrophoretic focusing and trapping of particles are both mitigated by $\mathrm{JH}$ effects. Moreover, this negative impact becomes more significant when dc-biased ac electric fields are applied rather than pure dc fields. We expect that the results obtained in this work will provide useful guidance for future designs of electrokinetic particle handling microdevices that will avoid $\mathrm{JH}$ effects or take advantage of them. A three-dimensional numerical model is currently under development for the purpose of understanding and accurately predicting $\mathrm{JH}$ and its effects on electroosmotic fluid flow and electrokinetic particle motion in constriction microchannels. 


\section{Acknowledgments}

This work is supported by NSF under grant no CBET-0853873 and by Clemson University through a start-up package to XX. The support from the National 973 Project (grant no 2011CB707604), NSFC (grant no 50890182) and Knowledge Innovation Program of CAS (KJCX2-YW-H18) to GH is gratefully appreciated as well. We also acknowledge the Open Fund support from LNM.

\section{References}

[1] Xuan X 2008 Electrophoresis 29 33-43

[2] Erickson D, Sinton D and Li D 2003 Lab on Chip 3 141-9

[3] Zhao T S and Liao Q 2002 J. Micromech. Microeng. 12 962-70

[4] Xuan X and Li D 2004 J. Micromech. Microeng. 14 1171-80

[5] Rathore A S 2004 J. Chromatogr. A 1037 431-43

[6] Huang K D and Yang R J 2006 Electrophoresis 27 1957-66

[7] Cetin B and Li D 2008 Electrophoresis 29 994-1005

[8] Hsieh S S and Yang T K 2008 J. Micromech. Microeng. 18025025

[9] Evenhuis C J and Haddad P R 2009 Electrophoresis 30 897-909

[10] Fu L M, Wang J H, Luo W B and Lin C H 2009 Microfluid. Nanofluid. 6 499-507

[11] Wong P K, Wang T, Deval J H and Ho C M 2004 IEEE/ASME Trans. Mechatronics 9 366-76

[12] Voldman J 2006 Annu. Rev. Biomed. Eng. 8 425-54

[13] Ai Y, Joo S, Jiang Y, Xuan X and Qian S 2009 Electrophoresis 30 2499-506

[14] Zhu J and Xuan X 2009 Electrophoresis 30 2668-75

[15] Kang K, Xuan X, Kang Y and Li D 2006 J. Appl. Phys. 99064702

[16] Pysher M D and Hayes M A 2007 Anal. Chem. 79 4552-7
[17] Ai Y, Qian S, Liu S and Joo S 2010 Biomicrofluidics 4013201

[18] Church C, Zhu J, Huang G, Tzeng T and Xuan X 2010 Biomicrofluidics 4044101

[19] Lewpiriyawong N, Yang C and Lam Y C 2012 Microfluid. Nanofluid. 12 723-33

[20] Braff W A, Pignier A and Buie C R 2012 Lab on Chip 12 1327-31

[21] Hawkins B G, Smith A E, Syed Y A and Kirby B J 2007 Anal. Chem. 79 7291-300

[22] Kang Y, Li D, Kalams S A and Eid J E 2008 Biomed. Microdevices 10 243-9

[23] Lewpiriyawong N, Yang C and Lam Y C 2008 Biomicrofluidics 2034105

[24] Jones P V, Staton S G R and Hayes M A 2011 Anal. Bioanal. Chem. 201 2103-11

[25] Srivastava S K, Gencoglu A and Minerick A R 2010 Anal. Bioanal. Chem. 399 301-21

[26] Regtmeier J, Eichhorn R, Viefhues M, Bogunovic L and Anselmetti D 2011 Electrophoresis 32 2253-73

[27] Hawkins B G and Kirby B J 2010 Electrophoresis 31 3622-33

[28] Sridharan S, Zhu J, Hu G and Xuan X 2011 Electrophoresis 32 2274-81

[29] Ross D and Locascio L E 2002 Anal. Chem. 74 2556-64

[30] Sommer G J, Kim S M, Littrell R J and Hasselbrink E F 2007 Lab on Chip 7 898-907

[31] Ge Z, Yang C and Tang G 2010 Int. J. Heat Mass Transfer $532722-31$

[32] Ge Z, Wang W and Yang C 2011 Lab on Chip 11 1396-402

[33] Xuan X, Xu B, Sinton D and Li D 2004 Lap on Chip 4 230-6

[34] Tang G, Yan D, Yang C, Gong H, Chai C and Lam Y 2006 Electrophoresis 27 628-39

[35] Ramos A, Morgan H, Green N G and Castellanos A 1998 J. Phys. D: Appl. Phys. 31 2338-53

[36] Xuan X and Li D 2005 J. Colloid Interface Sci. 289 291-303

[37] Kirby B J and Hasselbrink E F Jr 2004 Electrophoresis $25203-13$

[38] Zhu J, Canter R C, Keten G, Vedantam P, Tzeng T and Xuan X 2011 Microfluid. Nanofluid. 11 743-52 